\title{
Gastrointestinal comorbidities associated with atrial fibrillation
}

\author{
François Laliberté ${ }^{1}$, Yuliya Moore ${ }^{1}$, Katherine Dea', Joyce C LaMori ${ }^{2}$, Samir H Mody², JaCinda L Jones², \\ Michele D Arledge ${ }^{2}$, C V Damaraju ${ }^{2}$, Jeff R Schein ${ }^{2}$ and Patrick Lefebvre ${ }^{1}$
}

\begin{abstract}
This observational study was conducted to describe the risk of gastrointestinal $(\mathrm{Gl})$ events among patients with atrial fibrillation (AF). We analyzed Thomson Reuters MarketScan ${ }^{\circledast}$ data from 2005 to 2009. Subjects aged $\geq 18$ years with $\geq 1$ AF diagnosis were selected. Gl events were identified from claims with a primary or secondary diagnosis code for any Gl condition. The risk of Gl events was assessed using cumulative incidence (new Gl events/patients with AF without GI condition at baseline) and incidence rates (IRs), calculated as the number of patients with new Gl events divided by patient-years of observation. In addition, the $\mathrm{CHADS}_{2}$ score was evaluated at baseline to determine the patient's risk of stroke. A total of 557,123 AF patients were identified. The mean (median) AF patient age was 68.2 years (70); $45 \%$ were female. The cumulative incidences of any $\mathrm{Gl}$ event and dyspepsia were $40 \%$ and 19\%, respectively. The corresponding IRs were 38.8 and 14.7 events per 100 patient-years. IRs of any GI events for female and male patients were 43.6 and 35.5 ; for patients in the age groups $<65,65-74,75-84$, and $\geq 85$ years, IRs were 32.3, 38.9, 44.6, and 52.7; for patients with a $\mathrm{CHADS}_{2}$ score of 0, 1-2, 3-4, and 5-6, IRs were 30.3, 41.6, 56.9, and 74.5 , respectively. In this large claims database, $40 \%$ of AF patients experienced a Gl event, predominantly dyspepsia. Physicians should take age and comorbidities into consideration when managing AF patients.
\end{abstract}

Keywords: Atrial fibrillation; Risk; Gastrointestinal conditions; Dyspepsia

\section{Introduction}

Atrial fibrillation (AF) is the most common clinical arrhythmia; an estimated 2.3 million Americans were suffering from this condition in 2010 (Fuster et al. 2001; Go et al. 2001). AF is also strongly age dependent, affecting approximately $11-12 \%$ of persons $\geq 80$ years of age, compared with only $0.1-0.2 \%$ of persons $\leq 55$ years of age (Go et al. 2001). AF is commonly associated with other cardiovascular diseases, hypertension, including congestive heart failure, valvular heart disease, and ischemic heart disease (Lloyd-Jones et al. 2004). However, while literature documenting cardiovascular comorbidities is plentiful, less attention has been given to the prevalence and impact of gastrointestinal (GI) conditions such as dyspepsia, gastroesophageal reflux disease (GERD), peptic ulcer diseases, and GI bleeding in patients with AF (Hernandez-Diaz \& Rodriguez 2002; Locke et al. 1997; Talley et al. 1992; Talley et al. 1995). The GI tract has been documented as one of

\footnotetext{
* Correspondence: JLamori@its.jnj.com

2Janssen Scientific Affairs, LLC, Raritan, NJ, USA

Full list of author information is available at the end of the article
}

the most common locations of major bleeds attributed to a typical thromboprophylaxis regimen in stroke prevention (Coleman et al. 2012).

The number of GI conditions also increases with age (Hernandez-Diaz \& Rodriguez 2002; Blachut et al. 2004; Garcia Rodriguez et al. 1998; Som et al. 2010; Sostres et al. 2010). Dyspepsia, for example, is a common condition in the elderly. It is also a likely comorbidity in patients with AF. In a recent retrospective observational study, subjects with AF presenting with dyspepsia tended to have a greater health burden and lower quality of life than those without dyspepsia. Moreover, these patients were at greater risk of stroke (Lamori et al. 2012). The agents used in patients with AF to prevent stroke or treat other comorbidities are known to increase the risk of GI events. These agents include, but are not limited to, anticoagulants, nonsteroidal anti-inflammatory drugs (NSAIDs) (e.g. aspirin), corticosteroids, and calcium channel blockers (Garcia Rodriguez et al. 1998; Bytzer 2010). Agents currently used to treat patients with GI conditions

\section{实}


or to counteract treatment-induced GI events typically include acid secretory inhibitors, such as proton pump inhibitors (PPIs) (Bytzer 2010; McGowan et al. 2008; Yeomans et al. 1998).

GI conditions, in particular GERD, also have been proposed as a potential independent trigger for AF, because of the close anatomical positioning of the esophagus and the atria, and their similar nerve innervations (i.e. vagal nerve innervation) (Huang et al. 2012). The fact that vagal nerve overstimulation has been observed in patients with GERD and has been suggested as a contributing factor in AF supports the notion of GERD-mediated AF stimulation via vagal innervation. The most compelling evidence in support of GERD-mediated AF stimulation was found in a recent nationwide population-based survey in Taiwan, where GERD was reported to be independently associated with an increased risk of developing concomitant AF (Huang et al. 2012). It is thought that the prevalence of GERD increases with age. Whether this is the case has not yet been fully elucidated; nevertheless, esophageal symptoms (i.e. severe reflux esophagitis) have been reported to be more severe in older patients (Becher \& El Serag 2011).

In the US, the management of AF is dictated by guidelines issued by the American College of Chest Physicians, which use the $\mathrm{CHADS}_{2}$ classification to estimate stroke risk. This is established by adding points relating to risk factors of stroke - Congestive heart failure, Hypertension, Age $\geq 75$ years, Diabetes mellitus, prior Stroke or transient ischemic attack or thromboembolism: a higher score denotes a greater risk (Gage et al. 2004; Singer et al. 2008). Oral anticoagulant therapy is recommended for patients with a $\mathrm{CHADS}_{2}$ score $\geq 2$, while either warfarin or aspirin is recommended for patients with a $\mathrm{CHADS}_{2}$ score of 1 (Singer et al. 2008).

In light of the prevalence of GI comorbidities in patients with AF and to better understand how it affects this population, we conducted an observational study to document the extent of GI comorbidities in patients diagnosed with AF.

\section{Methods}

\section{Data source}

Health insurance claims from the Thomson Reuters MarketScan ${ }^{\odot}$ database were used to conduct the analysis. The MarketScan database, which combines two separate databases (Commercial Claims and Encounters and Medicare Supplemental and Coordination of Benefits $[\mathrm{COB}])$ to cover all age groups, contains claims from $\sim 100$ employers, health plans, and government and public organizations representing about 30 million covered lives. All US census regions are represented, with the South and North Central (Midwest) regions predominating. The MarketScan data used in the current analysis covered the period from January 2005 through December 2009.
Data used in the present study included health plan enrollment records, patient demographics, inpatient and outpatient medical services, and outpatient prescription drug dispensing records. Data included in the MarketScan database are de-identified and are in compliance with the Health Insurance Portability and Accountability Act (HIPAA) of 1996 to preserve patient anonymity and confidentiality.

\section{Study design}

A retrospective longitudinal cohort design was employed. To be included in the study sample, patients were required to meet the following criteria: (i) have at least one primary or secondary diagnosis of AF (ICD-9-CM code 427.31), (ii) have continuous health plan enrollment during the study period, and (iii) be at least 18 years of age as of the date of the index AF (index date). In addition, patients were required to have continuous health plan enrollment for at least 180 days prior to the index date (baseline/washout period). The observation period of patients spanned from the index date through the earlier of either the health plan disenrollment date or the end of data availability.

\section{Outcome measures}

The main endpoint of the study included the risk of GI events. These were defined as a primary or secondary diagnosis code for any GI event (see Additional file 1 for a complete list of ICD-9-CM codes) and the subset of GI events based on the classification in the recent Randomized Evaluation of Long-Term Anticoagulation (RE-LY) study (Connolly et al. 2009), including dyspepsia (including upper abdominal pain, abdominal pain, and abdominal discomfort, as well as dyspepsia), diarrhea, vomiting, and GI bleeding.

The secondary endpoints of the study included the following GI conditions: constipation, intestinal diverticula, dysphagia, esophagitis, flatulence, eructation and gas pain, gastritis and duodenitis, GERD, malignant neoplasm of the digestive organs and peritoneum, nausea alone, non-infectious gastroenteritis and colitis, other disorders of the intestine, and peptic ulcer diseases. GIrelated hospitalization was also reported; this was defined as a hospitalization that had any GI-related ICD-9-CM code associated with it, either as a primary or secondary diagnosis.

Rates of GI events were also calculated among subgroups with respect to gender, age ( $<65$ years, 65-74 years, 75-84 years, and $\geq 85)$, and $\mathrm{CHADS}_{2}$ score $(0$, $1-2,3-4$, and 5-6). The $\mathrm{CHADS}_{2}$ score consisted of the following stroke risk factors evaluated at baseline: congestive heart failure, hypertension, age $\geq 75$, diabetes, and prior stroke or transient ischemic attack ( 2 points for 
prior stroke or transient ischemic attack and 1 point each for other factors) (Singer et al. 2008).

\section{Statistical analyses}

Descriptive statistics were used to describe patient baseline characteristics. Means and standard deviations (SDs) were used to describe continuous variables; frequencies and percentages were reported for categorical variables.

The prevalence of GI events was calculated as the number of patients with a GI event during the 180-day baseline and/or study follow-up period divided by the total number of AF patients. Cumulative incidence, calculated as the number of patients with a new GI event (i.e. post-index AF diagnosis only) divided by the total number of AF patients without a history of GI events at baseline, was also reported. The 95\% confidence intervals (CIs) of the prevalence and cumulative incidence of GI events were computed using binomial distribution.

Finally, the incidence rates (IRs) of GI events were calculated as the number of new GI cases divided by patient-years of observation, which was censored at the time of the first event. This person-time approach is used to account for different lengths of observation among study subjects in a non-experimental setting. IR was expressed as number of new cases per 100 patients per year. The 95\% CIs of the IRs of GI events were computed using the Poisson distribution. All statistical analyses were performed using SAS version 9.2 (SAS Institute, Inc., Cary, NC).

\section{Results}

\section{Patient characteristics}

A total of 557,123 patients with AF met the inclusion criteria and formed the study population, of whom 143,955 (25.8\%) had a history of GI conditions during the 180day baseline period. Table 1 describes the baseline characteristics of the study population. The mean age (median; SD) was $68.2(70 ; 14.9)$ years, and 249,331 patients $(44.8 \%)$ were female. $\mathrm{CHADS}_{2}$ scores at baseline were 0, 1-2, 3-4, and 5-6, for 165,936 (29.8\%), 318,530 (57.2\%), 66,436 (11.9\%), and 6,221 (1.1\%) patients, respectively. Comorbidities at baseline included cardiovascular diseases (53.1\%), hypertension $(39.3 \%)$, diabetes $(20.0 \%)$, cancer $(13.5 \%)$, arthritis (12.7\%), and chronic kidney disease (6.5\%).

Medications that may cause GI events were taken by 359,398 (64.5\%) patients with AF at baseline, the most frequent (>10\%) drug classes being antibiotics (27.6\%), opioid pain medications (24.1\%), calcium channel blockers $(19.3 \%)$, anticoagulants $(18.9 \%)$, non-steroidal antiinflammatory drugs (NSAIDs; $12.1 \%$ ), selective serotonin reuptake inhibitors (SSRIs; 10.3\%), and corticosteroids (10.4\%).

Medications used to treat GI events were taken by 162,016 (29.1\%) patients with AF, among whom 110,762
(19.9\%) used PPIs, 24,122 used laxatives (4.3\%), 22,720 used H-2 antagonists (4.1\%), 19,550 used gastrointestinal medications (3.5\%), 14,165 used antiemetics (2.5\%), 6,895 used antidiarrheals (1.2\%), 1,198 used digestive aids $(0.2 \%)$, and 124 used antacids $(0.0 \%)$.

\section{Treatment patterns}

Table 2 presents the treatment patterns of medications associated with GI conditions during the observation period. The mean $( \pm S D)$ observation period for patients with AF was $543 \pm 455$ days (Table 1 ). During the followup, 398,633 (71.6\%) patients took at least one medication that may cause GI events: anticoagulant and antiplatelet agents were taken by $37.5 \%$ and $12.0 \%$ of patients with AF, respectively, whereas $225,833(40.5 \%)$ patients took at least one medication used to treat GI events (Table 2).The mean $( \pm \mathrm{SD})$ exposures to medications that may cause GI events and to medications used to treat GI events were $524 \pm 453$ and $393 \pm 410$ days, respectively (Table 2).

\section{Risk of Gl events}

Table 3 presents the prevalence and cumulative incidence of GI events. Over the 180-day baseline and mean follow-up of 543 days, 308,823 (55.4\%) patients had at least one GI event, 215,942 (38.8\%) had at least one GI event based on the RE-LY study classification, and 121,189 patients $(21.8 \%)$ had at least one GI-related hospitalization. Dyspepsia was the most common GI event, occurring in $29.6 \%$ of AF patients. The other most frequent GI events $(\geq 5 \%)$ included intestinal diverticula $(n=62,638$; 11.2\%), GERD ( $\mathrm{n}=63,159 ; 11.3 \%)$, GI bleeding $(\mathrm{n}=52,979$; $9.5 \%)$, other disorders of the intestine ( $\mathrm{n}=49,736 ; 8.9 \%)$, vomiting ( $\mathrm{n}=46,866,8.4 \%)$, gastritis and duodenitis ( $n=46,974 ; 8.4 \%)$, dysphagia $(n=46,506 ; 8.3 \%)$, diarrhea ( $n=43,628 ; 7.8 \%)$, constipation $(n=35,832 ; 6.4 \%)$, noninfectious gastroenteritis and colitis $(\mathrm{n}=29,602 ; 5.3 \%)$, and esophagitis $(\mathrm{n}=28,092 ; 5.0 \%)$.

\section{AF patients without a history of GI conditions at baseline}

For AF patients without a history of GI conditions at baseline, the cumulative incidences of any GI event, any GI event based on the RE-LY study classification, and dyspepsia were $39.9 \%, 26.3 \%$, and $19.1 \%$, respectively. The corresponding IRs were 38.8, 21.7, and 14.7 events per 100 patient-years, respectively (Figure 1). The IRs of any GI event for female and male patients were 43.6 and 35.5 , respectively (Figure 2). The IRs of any GI event increased with age and $\mathrm{CHADS}_{2}$ score: for patients in the age groups $<65,65-74,75-84$, and $\geq 85$ years, IRs were $32.3,38.9,44.6$, and 52.7 , respectively; for patients with a $\mathrm{CHADS}_{2}$ score of $0,1-2,3-4$, and 5-6, IRs were 30.3, $41.6,56.9$, and 74.5 , respectively. 
Table 1 Patient characteristics of the AF study population

\begin{tabular}{|c|c|c|}
\hline Variable & $\begin{array}{l}\text { All AF patients } \\
\text { ( } N=557,123)\end{array}$ & $\begin{array}{l}\text { AF patients witl } \\
\text { history of } \mathrm{Gl} \text { at } \\
\text { baseline }(\mathrm{N}=41\end{array}$ \\
\hline $\begin{array}{l}\text { Mean follow-up period, } \\
\text { days (SD) }\end{array}$ & $543.2(455.0)$ & $563.3(457.9)$ \\
\hline \multicolumn{3}{|l|}{ Demographics } \\
\hline $\begin{array}{l}\text { Age, years, mean (SD) } \\
\text { [median] }\end{array}$ & $68.2(14.9)[70]$ & $67.7(15)[69]$ \\
\hline Female, n (\%) & $249,331(44.8 \%)$ & $177,888(43.1 \%)$ \\
\hline \multicolumn{3}{|l|}{ Region, n (\%) } \\
\hline South & $210,204(37.7 \%)$ & $155,089(37.5 \%)$ \\
\hline West & $93,857(16.8 \%)$ & $71,646(17.3 \%)$ \\
\hline North Central & $179,174(32.2 \%)$ & $131,464(31.8 \%)$ \\
\hline Northeast & $72,331(13.0 \%)$ & $53,792(13.0 \%)$ \\
\hline Unknown & $1,557(0.3 \%)$ & $1,177(0.3 \%)$ \\
\hline \multicolumn{3}{|l|}{ Type of insurance, n (\%) } \\
\hline Comprehensive & $177,702(31.9 \%)$ & $129,040(31.2 \%)$ \\
\hline EPO & $1,335(0.2 \%)$ & $1,076(0.3 \%)$ \\
\hline HMO & $69,097(12.4 \%)$ & $53,120(12.9 \%)$ \\
\hline POS & $26,145(4.7 \%)$ & $19,932(4.8 \%)$ \\
\hline PPO & $259,366(46.6 \%)$ & $192,305(46.5 \%)$ \\
\hline POS with capitation & $2,308(0.4 \%)$ & $1,808(0.4 \%)$ \\
\hline CDHP & $4,567(0.8 \%)$ & $3,689(0.9 \%)$ \\
\hline Other/Unknown & 16,603 (3.0\%) & $12,198(3.0 \%)$ \\
\hline \multicolumn{3}{|l|}{ Year of index date, n (\%) } \\
\hline 2005 & $81,555(14.6 \%)$ & $62,282(15.1 \%)$ \\
\hline 2006 & $105,630(19.0 \%)$ & $79,372(19.2 \%)$ \\
\hline 2007 & $108,170(19.4 \%)$ & $80,423(19.5 \%)$ \\
\hline 2008 & $123,788(22.2 \%)$ & $91,663(22.2 \%)$ \\
\hline 2009 & $137,980(24.8 \%)$ & $99,428(24.1 \%)$ \\
\hline $\begin{array}{l}\text { Charlson comorbidity indexa } \\
\text { mean (SD) }\end{array}$ & $1.36(1.93)$ & $1.05(1.57)$ \\
\hline \multicolumn{3}{|l|}{$\mathrm{CHADS}_{2}^{\mathrm{a}, \mathrm{b}}$ score, $\mathrm{n}(\%)$} \\
\hline 0 & $165,936(29.8 \%)$ & 134,997 (32.7\%) \\
\hline $1-2$ & $318,530(57.2 \%)$ & $233,956(56.6 \%)$ \\
\hline $3-4$ & $66,436(11.9 \%)$ & $41,209(10.0 \%)$ \\
\hline $5-6$ & $6,221(1.1 \%)$ & $3,006(0.7 \%)$ \\
\hline \multicolumn{3}{|l|}{ History of $\mathrm{Gl}^{\mathrm{a}}$, n (\%) } \\
\hline Any Gl condition & $143,955(25.8 \%)$ & $0(0.0 \%)$ \\
\hline $\begin{array}{l}\text { Gl events based on the } \\
\text { RE-LY study classification }\end{array}$ & $87,017(15.6 \%)$ & $0(0.0 \%)$ \\
\hline Dyspepsia $^{d}$ & $64,202(11.5 \%)$ & $0(0.0 \%)$ \\
\hline Diarrhea & $12,693(2.3 \%)$ & $0(0.0 \%)$ \\
\hline Vomiting & $15,448(2.8 \%)$ & $0(0.0 \%)$ \\
\hline Gastrointestinal bleeding & $15,466(2.8 \%)$ & $0(0.0 \%)$ \\
\hline \multicolumn{3}{|l|}{ Other Gl events } \\
\hline Gastroesophageal reflux & $21,373(3.8 \%)$ & $0(0.0 \%)$ \\
\hline
\end{tabular}

Table 1 Patient characteristics of the AF study population (Continued)

\begin{tabular}{|c|c|c|}
\hline Diverticula of intestine & $19,640(3.5 \%)$ & $0(0.0 \%)$ \\
\hline Other disorders of intestine & $15,008(2.7 \%)$ & $0(0.0 \%)$ \\
\hline Gastritis and duodenitis & $13,923(2.5 \%)$ & $0(0.0 \%)$ \\
\hline Dysphagia & $13,306(2.4 \%)$ & $0(0.0 \%)$ \\
\hline Constipation & $10,669(1.9 \%)$ & $0(0.0 \%)$ \\
\hline $\begin{array}{l}\text { Noninfectious gastroenteritis } \\
\text { and colitis }\end{array}$ & $9,166(1.6 \%)$ & $0(0.0 \%)$ \\
\hline Esophagitis & $9,220(1.7 \%)$ & $0(0.0 \%)$ \\
\hline Nausea Alone & $6,532(1.2 \%)$ & $0(0.0 \%)$ \\
\hline $\begin{array}{l}\text { Malignant neoplasm of } \\
\text { digestive organs and } \\
\text { peritoneum }\end{array}$ & $9,702(1.7 \%)$ & $0(0.0 \%)$ \\
\hline $\begin{array}{l}\text { Flatulence, eructation, and } \\
\text { gas pain }\end{array}$ & $4,868(0.9 \%)$ & $0(0.0 \%)$ \\
\hline Peptic ulcer diseases & $4,352(0.8 \%)$ & $0(0.0 \%)$ \\
\hline Any Gl-related hospitalization & $43,851(7.9 \%)$ & $0(0.0 \%)$ \\
\hline \multicolumn{3}{|l|}{ Other comorbidities ${ }^{a}$, n (\%) } \\
\hline Cardiovascular diseases & $295,617(53.1 \%)$ & $202,017(48.9 \%)$ \\
\hline Chronic kidney disease & $36,187(6.5 \%)$ & $20,521(5.0 \%)$ \\
\hline Diabetes & $111,423(20.0 \%)$ & $76,751(18.6 \%)$ \\
\hline Hypertension & 218,765 (39.3\%) & $148,707(36.0 \%)$ \\
\hline Arthritis & $70,882(12.7 \%)$ & $47,053(11.4 \%)$ \\
\hline Any cancer & $74,954(13.5 \%)$ & $42,829(10.4 \%)$ \\
\hline \multicolumn{3}{|l|}{ Baseline medication $^{\mathrm{a}}, \mathrm{n}(\%)$} \\
\hline $\begin{array}{l}\text { Medications that may cause } \\
\text { Gl events }\end{array}$ & $359,398(64.5 \%)$ & $257,357(62.3 \%)$ \\
\hline Anticoagulants & 105,367 (18.9\%) & 80,059 (19.4\%) \\
\hline Antiplatelets & $55,143(9.9 \%)$ & $37,887(9.2 \%)$ \\
\hline Corticosteroids & $58,065(10.4 \%)$ & $36,247(8.8 \%)$ \\
\hline NSAIDs & $67,179(12.1 \%)$ & $47,315(11.5 \%)$ \\
\hline SSRIS & $57,567(10.3 \%)$ & $37,959(9.2 \%)$ \\
\hline Calcium channel blockers & $107,587(19.3 \%)$ & $77,563(18.8 \%)$ \\
\hline Bisphosphonates & $35,491(6.4 \%)$ & $24,934(6.0 \%)$ \\
\hline Antibiotic & $154,004(27.6 \%)$ & $105,071(25.4 \%)$ \\
\hline Pain medications (opioids) & $134,460(24.1 \%)$ & $84,629(20.5 \%)$ \\
\hline Antineoplastic & $15,634(2.8 \%)$ & $8,978(2.2 \%)$ \\
\hline Anesthesia medication & $236(0.0 \%)$ & $119(0.0 \%)$ \\
\hline $\begin{array}{l}\text { Medications used to treat } \\
\text { poisonings }\end{array}$ & $145(0.0 \%)$ & $80(0.0 \%)$ \\
\hline Iron-related medication & $89(0.0 \%)$ & $44(0.0 \%)$ \\
\hline $\begin{array}{l}\text { Medications used to treat } \\
\text { Gl events }\end{array}$ & $162,016(29.1 \%)$ & $91,083(22.0 \%)$ \\
\hline Antacids & $124(0.0 \%)$ & $78(0.0 \%)$ \\
\hline Antidiarrheals & $6,895(1.2 \%)$ & $2,592(0.6 \%)$ \\
\hline Antiemetics & $14,165(2.5 \%)$ & $7,138(1.7 \%)$ \\
\hline Digestive aids & $1,198(0.2 \%)$ & $429(0.1 \%)$ \\
\hline Gastrointestinal medications & $19,550(3.5 \%)$ & $7,709(1.9 \%)$ \\
\hline
\end{tabular}


Table 1 Patient characteristics of the AF study population (Continued)

\begin{tabular}{lll}
\hline Laxatives & $24,122(4.3 \%)$ & $7,893(1.9 \%)$ \\
Ulcer drugs & $133,053(23.9 \%)$ & $76,924(18.6 \%)$ \\
Proton Pump Inhibitors & $110,762(19.9 \%)$ & $62,566(15.1 \%)$ \\
H-2 Antagonists & $22,720(4.1 \%)$ & $14,862(3.6 \%)$ \\
\hline
\end{tabular}

$A F$ atrial fibrillation, GI gastrointestinal, EPO Exclusive Provider Organization, $H M O$ health maintenance organization, $P O S$ point of service, $P P O$ preferred provider organization, CDHP Consumer Directed Health Plans, SSRI selective serotonin reuptake inhibitors, NSAIDs nonsteroidal anti-inflammatory drugs.

${ }^{a}$ Based on a baseline period of 180 days prior to index date.

${ }^{\mathrm{b}} \mathrm{CHADS}_{2}$ score was calculated as 1 point for congestive heart failure, hypertension, age $\geq 75$, and diabetes mellitus, and 2 points for prior stroke or transient ischemic attack (Source: Gage Circulation 2004).

Including dyspepsia, diarrhea, vomiting, and gastrointestinal bleeding. IIncluding abdominal pain upper, abdominal pain, abdominal discomfort, and dyspepsia.

\section{Discussion}

Our analysis of real-world data demonstrates that a large proportion of patients with AF are at high risk of GI events. GI events were observed in more than half of the study population, with a prevalence of 55.4 per 100 persons. Dyspepsia was the most common GI symptom, reported in 557,123 patients with AF $(29.6 \%$; 164,892/ $557,123)$, accounting for $54 \%$ of all $308,823 \mathrm{GI}$ events reported. Dyspepsia is regarded as a significant burden for AF patients (Lamori et al. 2012), and in several studies of patients treated with NSAIDs and aspirin, an important reason for discontinuing treatment (CAPRIE Steering Committee 1996; Cryer et al. 2011; Niculescu et al. 2009; Ofman et al. 2003; Peto et al. 1988; Saini et al. 2009; Tournoij et al. 2009). Other common GI effects included intestinal diverticula $(62,638), \operatorname{GERD}(63,159)$, and GI bleeding $(52,979)$. GERD was recently found to be both a trigger for $\mathrm{AF}$ and associated with its development (Huang et al. 2012).

Consistent with past studies of cardiovascular disorders associated with AF (Carroll \& Majeed 2001), our study found cardiovascular diseases and hypertension to be frequent at baseline. Medications that can elicit GI adverse effects, ranging from dyspepsia to GI bleeding, include aspirin, other antiplatelet medications, anticoagulants, antibiotics, corticosteroids, SSRIs, NSAIDs, bisphosphonates, opioids and pain medications, calcium channel blockers, and iron-related medications, which are used to treat cardiovascular disorders and other comorbidities (e.g. depression and arthritis) (Garcia Rodriguez et al. 1998; Sostres et al. 2010; Bytzer 2010; Ashberg et al. 2010; Diego et al. 2011; Gabriel et al. 1991). In the current study, the most commonly used medications reported at baseline in AF patients, with known GI adverse effects, included pain medications (opioids), antibiotics, calcium channel blockers, and anticoagulants; 359,398 patients (64.5\%) received at least one medication that may cause GI events, and that
Table 2 Treatment patterns of medications associated with $\mathrm{GI}$ conditions

\begin{tabular}{|c|c|c|}
\hline Variable & $\begin{array}{l}\text { All AF patients } \\
(\mathrm{N}=557,123)\end{array}$ & $\begin{array}{l}\text { AF patients without } \\
\text { history of } \mathrm{Gl} \text { at } \\
\text { baseline }(\mathrm{N}=413,168)\end{array}$ \\
\hline \multicolumn{3}{|l|}{$\begin{array}{l}\text { Medications associated with } \\
\text { Gl, n (\%) }\end{array}$} \\
\hline $\begin{array}{l}\text { Medications that may cause } \\
\text { Gl events }{ }^{\mathrm{a}}\end{array}$ & $398,633(71.6 \%)$ & $298,490(72.2 \%)$ \\
\hline $\begin{array}{l}\text { Medications used to treat Gl } \\
\text { events }^{\mathrm{b}}\end{array}$ & 225,833 (40.5\%) & 154,164 (37.3\%) \\
\hline \multicolumn{3}{|l|}{$\begin{array}{l}\text { Exposure to therapy }{ }^{c} \text {, days, } \\
\text { mean (SD) }\end{array}$} \\
\hline $\begin{array}{l}\text { Medications that may cause } \\
\text { Gl events }{ }^{\mathrm{a}}\end{array}$ & $523.7(452.7)$ & $534.8(456.5)$ \\
\hline $\begin{array}{l}\text { Medications used to treat Gl } \\
\text { events }^{\mathrm{b}}\end{array}$ & $392.6(409.6)$ & $383.1(408.6)$ \\
\hline \multicolumn{3}{|l|}{$\begin{array}{l}\text { Dispensings per patient, } \\
\text { mean (SD) }\end{array}$} \\
\hline $\begin{array}{l}\text { Medications that may cause } \\
\text { Gl events }^{\mathrm{a}}\end{array}$ & $17.6(21.2)$ & 17.4 (20.8) \\
\hline $\begin{array}{l}\text { Medications used to treat Gl } \\
\text { events }^{\mathrm{b}}\end{array}$ & $7.4(9.3)$ & $7.0(8.8)$ \\
\hline \multicolumn{3}{|l|}{$\begin{array}{l}\text { Days of supply per dispensing } \\
\text { per patient, mean (SD) }\end{array}$} \\
\hline $\begin{array}{l}\text { Medications that may cause } \\
\text { Gl events }{ }^{\mathrm{a}}\end{array}$ & 34.7 (22.3) & $35.5(22.8)$ \\
\hline $\begin{array}{l}\text { Medications used to treat Gl } \\
\text { events }^{\mathrm{b}}\end{array}$ & 43.4 (28.6) & $43.0(29.2)$ \\
\hline \multicolumn{3}{|l|}{$\begin{array}{l}\text { Anticoagulant and } \\
\text { antiplatelet agents use, n (\%) }\end{array}$} \\
\hline Antiplatelet & $66,790(12.0 \%)$ & $48,808(11.8 \%)$ \\
\hline Anticoagulant & 208,985 (37.5\%) & 162,597 (39.4\%) \\
\hline
\end{tabular}

$A F$ atrial fibrillation, $G /$ gastrointestinal.

ancluding anticoagulant, antiplatelet, corticosteroids, NSAIDs, SSRIs, calcium channel blockers, bisphosponates, antibiotic, pain medications (opioids), antineoplastic, anesthesia medication, medications used to treat poisonings, and iron-related medication.

bIncluding antacids, antidiarrheals, antiemetics, digestive aids, gastrointestinal agents, laxatives, and ulcer drugs.

'Time from the date of the first dispensing to the end of the days of supply for the last dispensing.

proportion rose to $71.6 \%$ after the index diagnosis of AF. This finding could partly explain why, over the entire study period, $55.4 \%$ of patients with AF had at least one GI event. Although a large proportion of patients presented with GI events in our study, only $40.5 \%$ of patients with $\mathrm{AF}$ received treatment to counteract these events compared to $29.1 \%$ at baseline, where $80 \%$ of patients treated used at least one ulcer drug (i.e., PPIs or $\mathrm{H}-2$ antagonists).

In our study, we assumed that a number of patients with AF would have received more than one medication that could cause a GI event. The use of multiple medications by older patients reflects the multiple comorbidities in this population (Hajjar et al. 2007) and can 
Table 3 Prevalence and cumulative incidence of GI events

\begin{tabular}{|c|c|c|c|c|}
\hline \multirow[t]{2}{*}{ Variable } & \multicolumn{2}{|c|}{ All patients with $\mathrm{AF}(\mathrm{N}=557,123)$} & \multicolumn{2}{|c|}{ AF patients without history of $\mathrm{Gl}$ at baseline $(\mathrm{N}=413,168)$} \\
\hline & $\begin{array}{l}\text { Number of } \\
\text { Gl cases }^{\mathrm{a}}\end{array}$ & $\begin{array}{l}\text { Prevalence of } \mathrm{Gl}(\mathrm{per} \\
100 \text { persons) }\left[95 \% \mathrm{Cl}^{\mathrm{b}}\right]\end{array}$ & $\begin{array}{l}\text { Number of incident } \\
\text { Gl cases }^{c}\end{array}$ & $\begin{array}{l}\text { Cumulative incidence } \\
\text { (per } 100 \text { persons) }\left[95 \% \mathrm{Cl}^{\mathrm{b}}\right]\end{array}$ \\
\hline Any Gl event & 308,823 & $55.4[55.3,55.6]$ & 164,868 & $39.9[39.8,40.1]$ \\
\hline Gl events based on the RE-LY study classification ${ }^{d}$ & 215,942 & $38.8[38.6,38.9]$ & 108564 & $26.3[26.1,26.4]$ \\
\hline Dyspepsia $^{e}$ & 164,892 & $29.6[29.5,29.7]$ & 79,069 & $19.1[19.0,19.3]$ \\
\hline Gastrointestinal bleeding & 52,979 & $9.5[9.4,9.6]$ & 26,067 & $6.3[6.2,6.4]$ \\
\hline Vomiting & 46,866 & $8.4[8.3,8.5]$ & 20,297 & $4.9[4.8,5.0]$ \\
\hline Diarrhea & 43,628 & $7.8[7.8,7.9]$ & 19,928 & $4.8[4.8,4.9]$ \\
\hline \multicolumn{5}{|l|}{ Other Gl events } \\
\hline Diverticula of intestine & 62,638 & $11.2[11.2,11.3]$ & 30,983 & $7.5[7.4,7.6]$ \\
\hline Gastroesophageal reflux disease & 63,159 & $11.3[11.3,11.4]$ & 28,938 & $7.0[6.9,7.1]$ \\
\hline Other disorders of intestine & 49,736 & $8.9[8.9,9.0]$ & 23,435 & $5.7[5.6,5.7]$ \\
\hline Dysphagia & 46,506 & $8.3[8.3,8.4]$ & 22,071 & $5.3[5.3,5.4]$ \\
\hline Gastritis and duodenitis & 46,974 & $8.4[8.4,8.5]$ & 21,422 & $5.2[5.1,5.3]$ \\
\hline Constipation & 35,832 & $6.4[6.4,6.5]$ & 16,729 & $4.0[4.0,4.1]$ \\
\hline Noninfectious gastroenteritis and colitis & 29,602 & $5.3[5.3,5.4]$ & 12,837 & $3.1[3.1,3.2]$ \\
\hline Esophagitis & 28,092 & $5.0[5.0,5.1]$ & 11,968 & $2.9[2.8,2.9]$ \\
\hline Nausea Alone & 22,673 & $4.1[4.0,4.1]$ & 9,962 & $2.4[2.4,2.5]$ \\
\hline $\begin{array}{l}\text { Malignant neoplasm of digestive organs } \\
\text { and peritoneum }\end{array}$ & 18,107 & $3.3[3.2,3.3]$ & 5,534 & $1.3[1.3,1.4]$ \\
\hline Flatulence, eructation and gas pain & 17,263 & $3.1[3.1,3.1]$ & 7,209 & $1.7[1.7,1.8]$ \\
\hline Peptic ulcer diseases & 13,914 & $2.5[2.5,2.5]$ & 6,311 & $1.5[1.5,1.6]$ \\
\hline Any GI-related hospitalization & 121,189 & $21.8[21.6,21.9]$ & 55,744 & $13.5[13.4,13.6]$ \\
\hline
\end{tabular}

$A F$ atrial fibrillation, $G$ l gastrointestinal.

ancluding Gl events observed during the 180-day baseline or study follow-up period.

${ }^{\mathrm{b}}$ The $95 \%$ confidence intervals of $\mathrm{Gl}$ events were computed using the binomial distribution.

'Including $\mathrm{Gl}$ events observed only during the study follow-up period (i.e., patients with history of Gl at baseline were excluded).

dIncluding dyspepsia, diarrhea, vomiting, and gastrointestinal bleeding.

e Defined as abdominal pain upper, abdominal pain, abdominal discomfort, and dyspepsia.

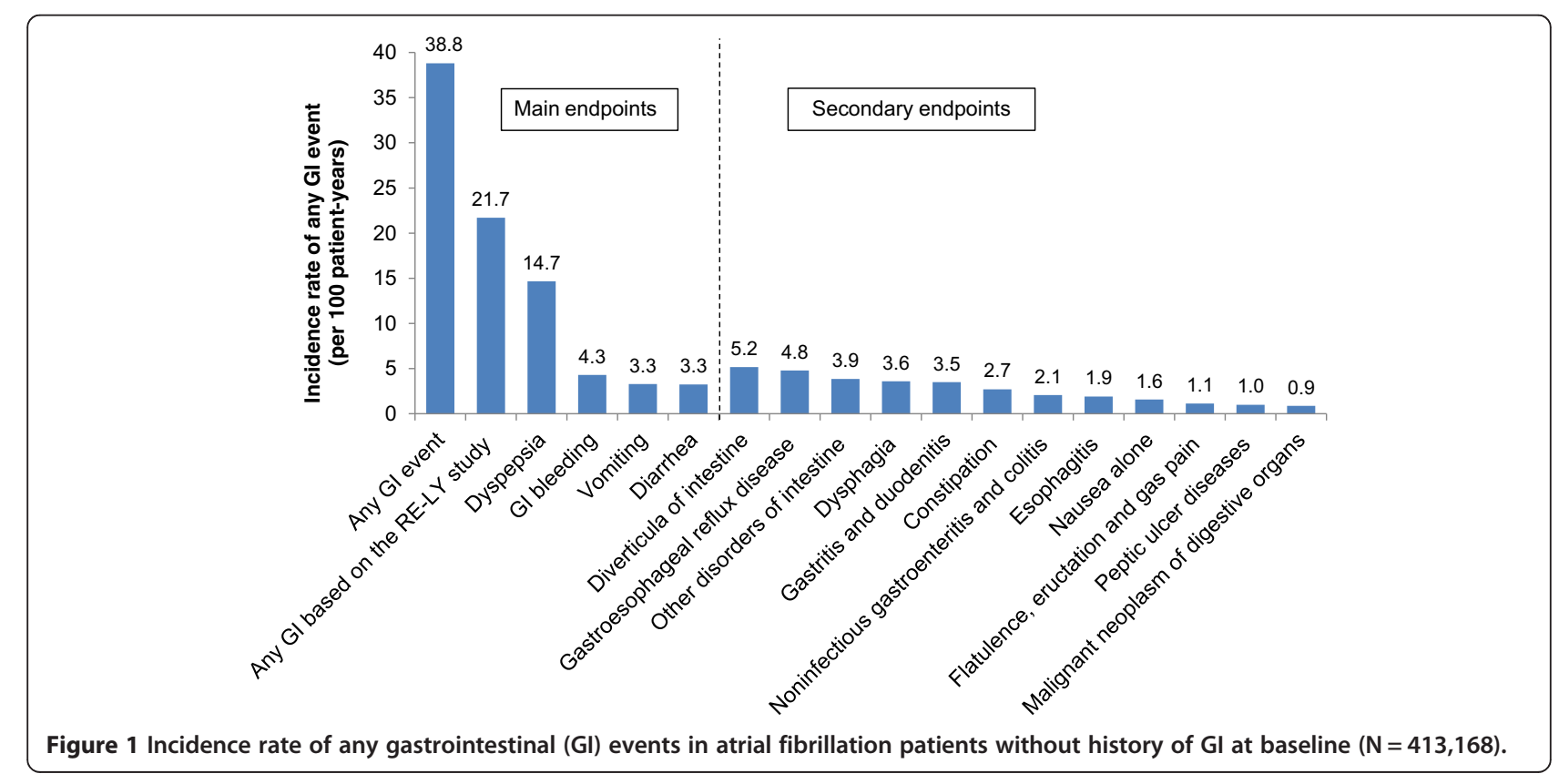




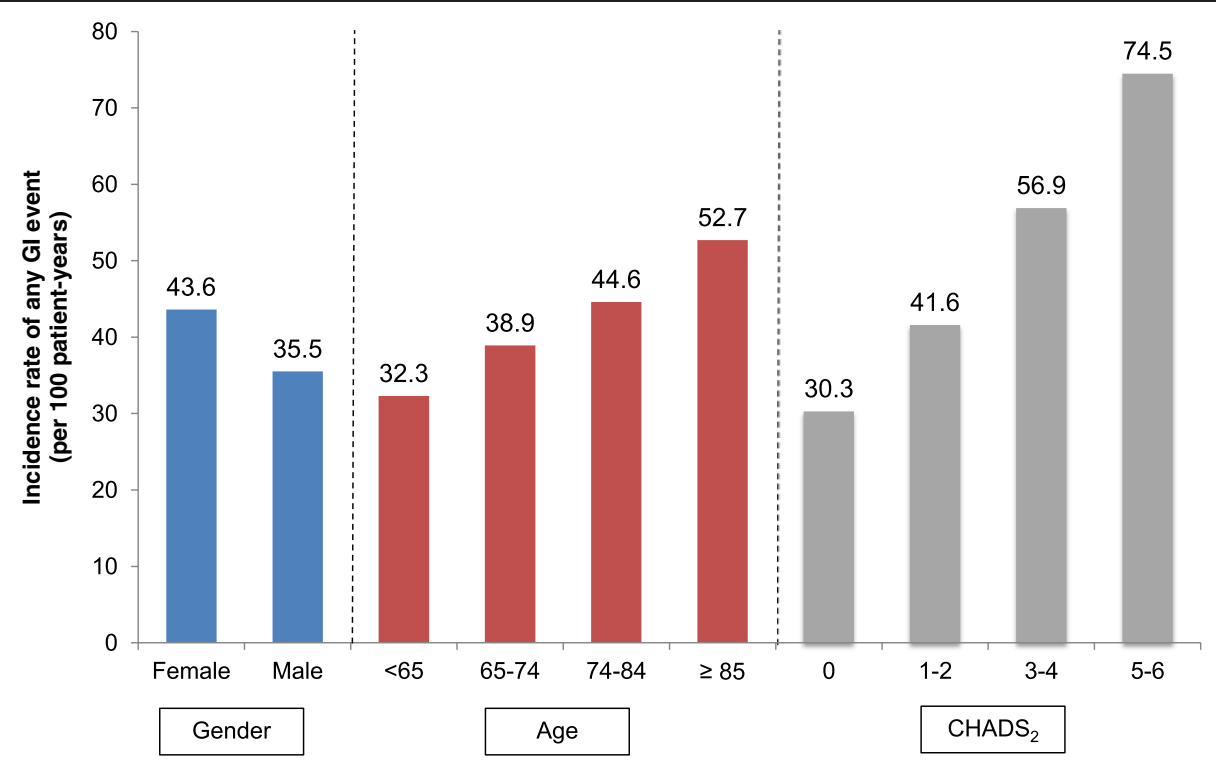

Figure 2 Incidence rate of any gastrointestinal (Gl) events stratified by gender, age, and $\mathrm{CHADS}_{2}$ (Congestive heart failure, Hypertension, Age $\geq 75$ years, Diabetes mellitus, prior Stroke or transient ischemic attack or thromboembolism) $(\mathrm{N}=413,168)$.

substantially increase their risk for GI events. For example, SSRIs increase the risk of GI bleeding up to three times and, when used concomitantly with NSAIDs, up to 15 times (Ashberg et al. 2010). Warfarin used concomitantly with aspirin, anti-infective agents, or NSAIDs, also has been shown to increase the risk of GI bleeding (Ashberg et al. 2010; Hallas et al. 2006; Man-Son-Hing \& Laupacis 2003; Schelleman et al. 2008; Shorr et al. 1993). Moreover, dual therapy in thromboprophylaxis has been found to increase patients' odds of experiencing a major GI bleed compared with monotherapy. Administering the antiplatelet agent clopidogrel with aspirin increased patients' odds of having a major GI bleed by 93\% compared with aspirin monotherapy (Coleman et al. 2012). Given the greater risk for stroke with older age, we may assume that patients in this age group are more likely to be candidates for dual thromboprophylaxis therapy and are therefore at greater risk for the subsequent GI effects attributed to this regimen.

Consistent with previous findings, in our study, advancing age was found to increase the risk of GI conditions, ranging from IRs of any GI event of 32.3 per 100 patient-years for patients aged $<65$ years to corresponding IRs of 52.7 per 100 patient-years for patients aged $\geq 85$ years. A higher $\mathrm{CHADS}_{2}$ score, indicative of greater comorbidity, also was associated with a higher risk of GI conditions, ranging from IRs of 30.3 per 100 patient-years for a $\mathrm{CHADS}_{2}$ score of 0 to corresponding IRs of 74.5 per 100 patient-years for a $\mathrm{CHADS}_{2}$ score of 5-6. Notably, subjects with higher $\mathrm{CHADS}_{2}$ scores tend to be older (i.e. $\geq 75$ years) (Oldgren et al. 2011). Given that the risk of GI events increases with age and that AF is strongly age dependent, this study highlights the importance of profiling the characteristics of patients with AF, in terms of both comorbidities and age, when making treatment decisions. We suggest that further research on GI adverse events in AF patients, specifically regarding the potential impact of AF therapy and age on GI conditions, is warranted. Moreover, the propensity for GI conditions, such as GERD, to trigger AF requires further elucidation. The possible impact of GI events and other comorbidities on the underuse of anticoagulants in AF patients also might be explored in future research.

Our study has a number of limitations. First, claims databases may contain inaccuracies or omissions in coded procedures, diagnoses, or pharmacy claims; however, it would be unlikely that these have significantly impacted our results considering the large sample size and the relatively high proportion of patients having a GI event in our study. Second, antiplatelet therapy was assessed based on pharmacy dispensing claims, and because the data do not capture nonprescription medications, such as aspirin, we may have underestimated antiplatelet utilization. Third, some medications used to treat GI conditions are also available without a prescription, which may further underestimate the utilization of these agents. In addition, the observational design was susceptible to various biases, such as information or classification bias (e.g. the identification of false positives of GI events). Despite these limitations, well-designed observational studies provide valuable information, with real-life scenarios and high generalizability. 


\section{Conclusion}

This large population-based study of more than 500,000 patients, based on real-world data, indicates a high risk of GI events, predominantly dyspepsia, among patients with AF. In view of the fact that GI comorbidities commonly coexist with AF, particularly in the elderly, it is important to take these comorbidities into account when managing AF.

\section{Additional file}

\section{Additional file 1: ICD-9-CM diagnosis codes used to identify} gastrointestinal conditions.

\section{Competing interests}

This research was funded by Janssen Scientific Affairs, LLC, Raritan, NJ, USA. Groupe d'analyse, Ltée was responsible for conducting the analyses. All authors have contributed to the elaboration of the study design and the methodology, the interpretation of the results, the development and writing of the draft manuscript, and have approved the current version. F.L., Y.M., K. D., and P.L. are employees of Groupe d'analyse, Ltée, a consulting company that has received research grants from Janssen Scientific Affairs, LLC, and six of the authors (J.C.L., S.H.M., J.L.J., M.D.A., C.V.D., and J.R.S.) are current or former employees of Janssen Scientific Affairs, LLC.

\section{Authors' contribution}

All authors have contributed to the elaboration of the study design and the methodology, the interpretation of the results, the development and writing of the draft manuscript, and have approved the current version.

\section{Acknowledgements}

The authors would like to acknowledge Victoria Panagakis, who provided editorial support with funding from Janssen Scientific Affairs, LLC. Parts of this manuscript were presented at the American Heart Association (AHA) Scientific Sessions 2011, November 12-16, Orlando, Florida, and the American College of Cardiology (ACC) Annual Scientific Session, March 24-27, 2012, Chicago, Illinois.

\section{Author details}

'Groupe d'analyse, Ltée, 1000 rue de la Gauchetière Ouest, Bureau 1200, Montréal, Québec H3B 4 W5, Canada. Janssen Scientific Affairs, LLC, Raritan, NJ, USA.

\section{Received: 1 April 2014 Accepted: 3 September 2014}

Published: 15 October 2014

\section{References}

Ashberg K, Hoglund P, Kim WH, von Holstein CS (2010) Impact of aspirin, NSAIDs, warfarin, corticosteroids and SSRIs on the site and outcome of non-variceal upper and lower gastrointestinal bleeding. Scand J Gastroenterol 45:1404-1415

Becher A, El Serag H (2011) Systematic review: the association between symptomatic response to proton pump inhibitors and health-related quality of life in patients with gastro-oesophageal reflux disease. Aliment Pharmacol Ther 34:618-627

Blachut K, Paradowski L, Garcarek J (2004) Prevalence and distribution of the colonic diverticulosis. Review of 417 cases from Lower Silesia in Poland. Rom J Gastroenterol 13:281-285

Bytzer P (2010) Dyspepsia as an adverse effect of drugs. Best Pract Res Clin Gastroenterol 24:109-120

CAPRIE Steering Committee (1996) A randomised, blinded, trial of clopidogrel versus aspirin in patients at risk of ischaemic events (CAPRIE). CAPRIE Steering Committee. Lancet 348:1329-1339

Carroll K, Majeed A (2001) Comorbidity associated with atrial fibrillation: a general practice-based study. Br J Gen Pract 51:884-891

Coleman Cl, Sobieraj DM, Winkler S, Cutting P, Mediouni M, Alikhanov S, Kluger J (2012) Effect of pharmacological therapies for stroke prevention on major gastrointestinal bleeding in patients with atrial fibrillation. Int J Clin Pract 66:53-63

Connolly SJ, Ezekowitz MD, Yusuf S, Eikelboom J, Oldgren J, Parekh A, Pogue J, Reilly PA, Themeles E, Varrone J, Wang S, Alings M, Xavier D, Zhu J, Diaz R, Lewis BS, Darius H, Diener HC, Joyner CD, Wallentin L, RE-LY Steering Committee and Investigators (2009) Dabigatran versus warfarin in patients with atrial fibrillation. N Engl J Med 361:1139-1151

Cryer B, Luo X, Assaf AR, Sands G, Mardekian J (2011) Persistence with nonselective NSAIDs and celecoxib among patients with gastroesophagea reflux disease and osteoarthritis or rheumatoid arthritis. Curr Med Res Opin 27:295-302

Diego L, Atayee R, Helmons P, Hsiao G, von Gunten CF (2011) Novel opioid antagonists for opioid-induced bowel dysfunction. Expert Opin Investig Drugs 20:1047-1056

Fuster V, Ryden LE, Asinger RW, Cannom DS, Crijns HJ, Frye RL, Halperin JL, Kay GN, Klein WW, Lévy S, McNamara RL, Prystowsky EN, Wann LS, Wyse DG, American College of Cardiology; American Heart Association; European Society of Cardiology; North American Society of Pacing and Electrophysiology (2001) ACC/AHA/ESC guidelines for the management of patients with atrial fibrillation. A report of the American College of Cardiology/American Heart Association Task Force on Practice Guidelines and the European Society of Cardiology Committee for Practice Guidelines and Policy Conferences (Committee to develop guidelines for the management of patients with atrial fibrillation) developed in collaboration with the North American Society of Pacing and Electrophysiology. Eur Heart J 22:1852-1923

Gabriel SE, Jaakkimainen L, Bombardier C (1991) Risk for serious gastrointestinal complications related to use of nonsteroidal anti-inflammatory drugs, A meta-analysis. Ann Intern Med 115:787-796

Gage BF, van Walraven C, Pearce L, Hart RG, Koudstaal PJ, Boode BS, Petersen P (2004) Selecting patients with atrial fibrillation for anticoagulation: stroke risk stratification in patients taking aspirin. Circulation 110:2287-2292

Garcia Rodriguez LA, Cattaruzzi C, Troncon MG, Agostinis L (1998) Risk of hospitalization for upper gastrointestinal tract bleeding associated with ketorolac, other nonsteroidal anti-inflammatory drugs, calcium antagonists, and other antihypertensive drugs. Arch Intern Med 158:33-39

Go AS, Hylek EM, Phillips KA, Chang Y, Henault LE, Selby JV, Singer DE (2001) Prevalence of diagnosed atrial fibrillation in adults: national implications for rhythm management and stroke prevention: the AnTicoagulation and Risk Factors in Atrial Fibrillation (ATRIA) Study. JAMA 285:2370-2375

Hajjar ER, Cafiero AC, Hanlon JT (2007) Polypharmacy in elderly patients. Am J Geriatr Pharmacother 5:345-351

Hallas J, Dall M, Andries A, Andersen BS, Aalykke C, Hansen JM, Andersen M, Lassen AT (2006) Use of single and combined antithrombotic therapy and risk of serious upper gastrointestinal bleeding: population based casecontrol study. BMJ 333:726

Hernandez-Diaz S, Rodriguez LA (2002) Incidence of serious upper gastrointestinal bleeding/perforation in the general population: review of epidemiologic studies. J Clin Epidemiol 55:157-163

Huang CC, Chan WL, Luo JC, Chen YC, Chen TJ, Chung CM, Huang PH, Lin SJ, Chen JW, Leu HB (2012) Gastroesophageal reflux disease and atrial fibrillation: a nationwide population-based study. PLoS One 7:e47575

Lamori JC, Mody SH, Gross HJ, DiBonaventura M, Patel A, Schein J, Nelson WW (2012) Dyspepsia and disease burden among patients with atrial fibrillation. Crit Pathw Cardiol 11:14-19

Lloyd-Jones DM, Wang TJ, Leip EP, Larson MG, Levy D, Vasan RS, D'Agostino RB, Massaro JM, Beiser A, Wolf PA, Benjamin EJ (2004) Lifetime risk for development of atrial fibrillation: the Framingham Heart Study. Circulation 110:1042-1046

Locke GR III, Talley NJ, Fett SL, Zinsmeister AR, Melton LJ III (1997) Prevalence and clinical spectrum of gastroesophageal reflux: a population-based study in Olmsted County, Minnesota. Gastroenterology 112:1448-1456

Man-Son-Hing M, Laupacis A (2003) Anticoagulant-related bleeding in older persons with atrial fibrillation: physicians' fears often unfounded. Arch Intern Med 163:1580-1586

McGowan B, Bennett K, Barry M, Canny M (2008) The utilisation and expenditure of medicines for the prophylaxis and treatment of osteoporosis. Ir Med J 101:38-41

Niculescu L, Li C, Huang J, Mallen S (2009) Pooled analysis of Gl tolerability of 21 randomized controlled trials of celecoxib and nonselective NSAIDs. Curr Med Res Opin 25:729-740 
Ofman JJ, Maclean CH, Straus WL, Morton SC, Berger ML, Roth EA, Shekelle PG (2003) Meta-analysis of dyspepsia and nonsteroidal antiinflammatory drugs. Arthritis Rheum 49:508-518

Oldgren J, Alings M, Darius H, Diener HC, Eikelboom J, Ezekowitz MD, Kamensky G, Reilly PA, Yang S, Yusuf S, Wallentin L, Connolly SJ, Investigators RE-LY (2011) Risks for stroke, bleeding, and death in patients with atrial fibrillation receiving dabigatran or warfarin in relation to the CHADS2 score: a subgroup analysis of the RE-LY trial. Ann Intern Med 155:660-667, W204

Peto R, Gray R, Collins R, Wheatley K, Hennekens C, Jamrozik K, Warlow C, Hafner B, Thompson E, Norton S, Gilliland J, Doll R (1988) Randomised trial of prophylactic daily aspirin in British male doctors. Br Med I (Clin Res Ed) 296:313-316

Saini SD, Schoenfeld P, Kaulback K, Dubinsky MC (2009) Effect of medication dosing frequency on adherence in chronic diseases. Am J Manag Care 15:e22-e33

Schelleman H, Bilker WB, Brensinger CM, Han X, Kimmel SE, Hennessy S (2008) Warfarin with fluoroquinolones, sulfonamides, or azole antifungals: interactions and the risk of hospitalization for gastrointestinal bleeding Clin Pharmacol Ther 84:581-588

Shorr RL, Ray WA, Daugherty JR, Griffin MR (1993) Concurrent use of nonsteroidal anti-inflammatory drugs and oral anticoagulants places elderly persons at high risk of hemorrhagic peptic ulcer disease. Ann Intern Med 153:1665-1670

Singer DE, Albers GW, Dalen JE, Fang MC, Go AS, Halperin JL, Lip GY, Manning WJ, American College of Chest Physicians (2008) Antithrombotic therapy in atrial fibrillation: American College of Chest Physicians Evidence-Based Clinical Practice Guidelines (8th Edition). Chest 133:546S-592S

Som R, Gossage JA, Crane A, Rowe PH (2010) Surgical workload, risk factors and complications in patients on warfarin with gastrointestinal bleeding. Int J Surg 8:52-55

Sostres C, Gargallo CJ, Arroyo MT, Lanas A (2010) Adverse effects of non-steroidal anti-inflammatory drugs (NSAIDs, aspirin and coxibs) on upper gastrointestinal tract. Best Pract Res Clin Gastroenterol 24:121-132

Talley NJ, Zinsmeister AR, Schleck CD, Melton LJ III (1992) Dyspepsia and dyspepsia subgroups: a population-based study. Gastroenterology 102:1259-1268

Talley NJ, Evans JM, Fleming KC, Harmsen WS, Zinsmeister AR, Melton L III (1995) Nonsteroidal antiinflammatory drugs and dyspepsia in the elderly. Dig Dis Sci 40:1345-1350

Tournoij E, Peters RJ, Langenberg M, Kanhai KJ, Moll FL (2009) The prevalence of intolerance for low-dose acetylsalicylacid in the secondary prevention of atherothrombosis. Eur J Vasc Endovasc Surg 37:597-603

Yeomans ND, Tulassay Z, Juhász L, Rácz I, Howard JM, van Rensburg CJ, Swannell AJ, Hawkey CJ (1998) A comparison of omeprazole with ranitidine for ulcers associated with nonsteroidal antiinflammatory drugs. Acid Suppression Trial: Ranitidine versus Omeprazole for NSAID-associated Ulcer Treatment (ASTRONAUT) Study Group. N Engl J Med 338:719-726

doi:10.1186/2193-1801-3-603

Cite this article as: Laliberté et al:: Gastrointestinal comorbidities associated with atrial fibrillation. SpringerPlus 2014 3:603.

\section{Submit your manuscript to a SpringerOpen ${ }^{\circ}$ journal and benefit from:}

- Convenient online submission

- Rigorous peer review

- Immediate publication on acceptance

- Open access: articles freely available online

- High visibility within the field

- Retaining the copyright to your article

Submit your next manuscript at $\gg$ springeropen.com 